\title{
Immune development in late preterm neonates
}

\author{
Gaetano Chirico \\ From XX National Congress of the Italian Society of Neonatology \\ Rome, Italy. 9-11 October 2014
}

Several recent studies have underlined that late preterm infants have a significantly increased risk of infection and sepsis, [1-3] mainly related to problems of adaptation from intra- to extra-uterine life of the immune defense mechanisms. Indeed, both the innate (natural, non specific) and the adaptive (acquired, specific) immune systems are incompletely developed at birth, the more preterm the neonate, the more severe and prolonged the immunodeficiency.[4,5]

$\mathrm{T}$ lymphocytes response to mitogens is poor, and $\mathrm{T}$ and $\mathrm{B}$ lymphocytes are immature: higher percentage of $\mathrm{CD} 4^{+}$ $\mathrm{T}$ lymphocytes and lower of $\mathrm{CD} 8^{+}$cells, with a gradual decline with age of the $\mathrm{CD} 4^{+} / \mathrm{CD} 8^{+}$ratio, and predominant naive phenotype with elevated percentages of CD4 ${ }^{+} / \mathrm{CD}_{4} 5 \mathrm{RA}^{+} \mathrm{T}$ cells; in addition, cytokine production is reduced and Th1-like response inadequate. The immaturity of lymphocytes and of antigen presenting cells are responsible for the marked deficiency of antibody production; also, levels of IgG are low in late preterm infants because transplacental passage from the mother mostly occurs during the last trimester of gestation; therefore, these neonates may lack the protection ensured by maternal derived pathogen-specific IgG. The inability to produce adequate amounts of hematopoietic growth factors, particularly G- and GM-CSF, and the reduced neutrophil, complement and natural killer cell activity, may further amplify the neonatal impairment of immune defenses.

The combined neonatal deficiency of immunoglobulin, complement and neutrophil activity results in increased susceptibility to systemic infections from encapsulated pathogens, such as Group B Streptococcus, Staphylococci and Klebsiella, that require opsonization for efficient phagocytosis and killing. The immaturity of pattern recognition receptors (PRR) response to pathogen-associated molecular patterns (PAMP), in particular the impaired TLR4 (Toll Like Receptor) signaling, [1] may contribute

Correspondence: gaechirico@alice.it

Neonatology and Neonatal Intensive Care Unit, Children Hospital, Spedali Civili, Brescia, Italy 